\title{
Assessment of pap smear and colposcopic findings in patients with vaginal discharge
}

\author{
Nagajan Bhadarka, Nimish Pandya, Satyam Joshi \\ Correspondence: Dr Nimish Pandya, Professor \& HOD, Department of Obstetrics \& \\ Gynaecology, Gujarat Adani Institute of Medical Science, Bhuj, Kutch, Gujarat, India; \\ Email - researchguide86@gmail.com \\ Distributed under Attribution-NonCommercial-ShareAlike 4.0 International (CC BY-NC-SA 4.0)
}

\begin{abstract}
Aim: Present study was performed with an aim to see the types of vaginal discharge with the pap smear and colposcopic findings. Material and Methods: This cross-sectional study was conducted in 68 women attending outpatient department (OPD) of Obstetrics and Gynaecology. Types of vaginal discharge with pap smear, colposcopy findings such as normal impression, inflammatory changes, cervical intraepithelial neoplasia and invasive carcinoma were observed. Results: Among the study group, majority of them $51.9 \%$ came to the hospital with history of vaginal discharge which is thick, white and $42.4 \%$ gave history of thin watery vaginal discharge. In the present study $14.5 \%$ gave history of post coital bleeding and $22.2 \%$ women gave history of post menopausal bleeding. Cervical biopsy was taken in all the patients, of which $15.7 \%$ had no evidence of malignancy, 30.9\% had CIN I, $16.10 \%$ had CIN II, 19.7\% had CIN III, $8.4 \%$ had CIS, $7.1 \%$ were found to have squamous cell carcinoma, $1.4 \%$ had adenocarcinoma. Conclusion: Carcinoma cervix has a long pre invasive state. Excessive vaginal discharge for long duration is to be assessed for cervical pathology.
\end{abstract}

Keywords: Carcinoma, colposcopy, pap smear, squamous cell carcinoma.

Cervix is the lower narrow portion of uterus with an external os and internal os. Squamocolumnar junction is the junction between the squamous and glandular epithelium and its location varies based on age, hormonal influence. In younger age columnar epithelium lies near external os. Later after puberty due to metaplasia, of columnar epithelium the squamous epithelium comes near external os. Transformation zone lies between this original and new squamocolumnar junction. Cervical pathology may be cervical erosion, cervicitis, tuberculosis of cervix, cervical intraepithelial neoplasia (CIN), and frank malignancy of cervix. Carcinoma is the most common gynecological growth in the developing countries and the third most frequently diagnosed cancer in Indian women. Cancer of the cervix is preventable, yet approximately 493,100 new cases and more than 273, 00 deaths each year among women worldwide.

While evidence of effective screening programs can be seen throughout the developed world's burden and impact of the disease remains high in the developing countries where $85 \%$ of disease related deaths occur. India, which accounts for one sixth of the world's population and bears one fifth of the world's burden of cervical cancer. There are approximately 130,000 new cases of cervical cancer in

Received: $20^{\text {th }}$ June 2018. Accepted: $25^{\text {th }}$ September 2018.

Bhadarka N, Pandya N, Joshi S. Assessment of pap smear and colposcopic findings in patients with vaginal discharge. The New Indian Journal of OBGYN. 2019; 5(2): 136-9 
India per year and the disease is reported to be responsible for $20 \%$ of all female deaths. India's age standardized incidence rate $(30.7$ per100, 000) and age standardized mortality rate are the highest in south East Asia. Global evidence demonstrates that the key to reducing cervical cancer morbidity and mortality is early detection coupled with timely treatment of cervical precancerous lesions. ${ }^{1-7}$

The concept of pre-invasive disease of cervix was introduced in 1947. This pathology can be diagnosed clinically, or even early by a simple inexpensive, noninvasive screening test called pap smear, and if needed colposcopy and correlated with cervical biopsy. Early intervention reduces the morbidity and reduces the mortality rates of carcinoma cervix. Colposcopy is an optical method of visualizing lower genital tract under bright illumination using stereoscopic vision. It is a simple non-invasive outpatient department procedure. Colposcopic directed biopsies of suspicious area provide the final confirmation of diagnosis in most of the situations. ${ }^{2-4}$ The present study was performed to see the types of vaginal discharge with pap smear and colposcopic findings in patients attending in tertiary care centre.

\section{Material and Methods}

Present cross-sectional study was conducted in 68 women attending outpatient department (OPD) of Obstetrics and Gynaecology, tertiary center of Bhuj, Kutch. An inclusion criterion for women was women in the age group of $37-54$ years. Written informed consent was obtained from the patient before enrolling them into study. A detailed history was taken with emphasis on age, parity, menstrual history for inter menstrual bleeding, excessive bleeding per vagina during menstruation, use of contraceptives, excessive white discharge with itching/ foul smelling,. Systemic examination followed by local examination, patient in dorsal position with clear illumination using Cusco's speculum. Cervix visualized for any local lesion and abnormal discharge. Pap smear was taken from squamocolumnar junction. The smear was air dried, smears hydrated and immersed in hematoxylin for 1-2mins and rinsed in tap water and stained with Papanicoloau stain. Pap smear cytological findings like normal cytology, cytology with inflammatory changes, cytology with atypical squamous cells and dysplastic cytology, colposcopy findings such as normal impression, inflammatory changes, cervical intraepithelial neoplasia and invasive carcinoma was observed. Colposcopic directed punch biopsy is taken and the tissue was sent for histopathological examination.

The data was coded and entered into Microsoft Excel spreadsheet. Analysis was done using SPSS version 15 (SPSS Inc. Chicago, IL, USA) windows software program. Descriptive statistics were calculated.

\section{Results}

Out of the 68 participants majority of them belong to the age group of $>51 \mathrm{yrs}$ (Table 1$)$. Maximum number of

Table 1: Distribution of number of patients according to Age

\begin{tabular}{ccc}
\hline Age & Number & Percentage $\%$ \\
\hline $41-51$ & 18 & 26.4 \\
$>51$ & 50 & 73.5 \\
Total & 68 & 100.0 \\
\hline
\end{tabular}

patients presenting with vaginal discharge were in the age group more than 51 years $(73.5 \%)$ and $26.4 \%$ were of 41 51 years. Among the study population, majority of women belonged to lower middle socioeconomic status $51.4 \%, 36.7 \%$ were of upper middle socioeconomic status (Table 2). Among the study group, most of them 51.9\% came to the hospital with history of vaginal discharge which is thick, white and $42.4 \%$ gave history of thin watery vaginal discharge. $5.7 \%$ had blood stained discharge. In the present study, $14.5 \%$ gave history of post coital bleeding and $22.2 \%$ women gave history of post menopausal bleeding. Among the study group,

Table 2: Distribution of number of patients according to socio economic status

\begin{tabular}{ccc}
\hline Socioeconomic Status & Number & Percentage \% \\
\hline Upper & 3 & 4.4 \\
Middle upper & 25 & 36.7 \\
Middle lower & 35 & 51.4 \\
Upper lower & 5 & 7.3 \\
Total & 68 & 100.0 \\
\hline
\end{tabular}

majority of them $84.9 \%$ had regular menstrual cycle, majority of them $52.7 \%$ married at age less than 20 years. In our study group, majority $83.9 \%$ were multiparous women. In the study group, most of them about $85.9 \%$ 
had no significant finding in per abdominal examination. Among the study population, majority $51.2 \%$ had mucopurulent discharge. $32.2 \%$ had thick curdy white discharge while $8.5 \%$ had blood stained discharge. In $94.9 \%$ women wet smear was not positive for any other organism. In our study, pap smear was taken for all patients of which $33.6 \%$ of smear were HSIL, $25.3 \%$ were LSIL, $21.3 \%$ came as inflammatory smear, $12.8 \%$ showed ASCUS, $8.6 \%$ were normal, 1.3\% showed adenocarcinoma. Cervical biopsy was taken in all the patients, of which $15.7 \%$ had no evidence of malignancy, $30.9 \%$ had CIN I, $16.10 \%$ had CIN II, 19.7\% had CIN III, $8.4 \%$ had CIS, $7.1 \%$ were found to have squamous cell carcinoma, $1.4 \%$ had adenocarcinoma.

\section{Discussion}

This study was conducted in 68 women attending OPD at department of Obstetrics and Gynaecology, tertiary center of Bhuj, Kutch. They were also subjected to colposcopy and biopsy was taken and the results of pap smear, colposcopic findings were observed in detecting pre cancerous lesions of cervix. Kushtagy et $\mathrm{al}^{8}$ in their study said that prevalence of CIN was higher in women aged more than 30 years. Based on the distribution of study population, according to socio economic status of women, majority of women with pre cancerous lesions belong to lower socio economic status. Vaidya et $\mathrm{al}^{9}$ had showed in his study that most of CIN I (80\%) and CIN II $(50 \%)$ were belonged to low socio economic status as poor personal hygiene adds to the risk of developing cervical cancer. Based on the presenting complaint, majority of the women had vaginal discharge which was thick as in $51.9 \%$ and thin watery in about $42.4 \%$ for a duration of more than 6 months. Excessive vaginal discharge for long duration has also been proven to be a risk factor for development of cervical cancer. About $14.5 \%$ of the study population had post coital bleeding, which has positive association with the development of CIN. Shalini et $\mathrm{al}^{10}$ exposed that among women with post coital bleeding 5.6\% had CIN I, 3.6\% had CIN II, III and $55 \%$ had invasive cancer. Women with post menopausal bleeding accounted for $22.2 \%$ in this study. Early age of marriage, multiple numbers of sexual partners and sex with high risk males whose wives died due to cervical cancer also increase the risk of development of CIN. Mayavati et $\mathrm{al}^{11}$ in her study showed the relationship of cervical cancer with early marriage. In our study among women who had CIN majority were married at age less than 20 years. Among women who were permanently sterilized $39.2 \%$ had CIN. Stern et al $^{12}$ proposed that there is an increased risk of progression to cervical dysplasia among the hormonal contraceptive users. They also stated that the risk of CIN increases up to 4 fold in HPV positive women taking oral contraceptive pills.

In our study, erosion which is the most common finding was seen in about $76 \%$ (54/71) women among them 74\% (40/54) had CIN. Hypertrophied cervix with no erosion was seen in about $15.4 \%$ of study population among which $63 \%$ were found to have CIN. Pap smear was taken in all the patients and showed LSIL in $25.3 \%$ of women and HSIL $33.6 \%$ of study population. Accuracy of detecting pre cancerous lesions was calculated and was found that accuracy in detecting pre cancerous lesions by colposcopy $85.9 \%$ is higher than by Pap smear $46.4 \%$. Massad et $\mathrm{al}^{13}$ in his study reported that accuracy in detecting CIN by colposcopy is about $80 \%$. Olanian et $\mathrm{al}^{14}$ reported that accuracy of colposcopic detection was about $89 \%$.

\section{Conclusion}

Carcinoma cervix is painstaking to be preventive disease as it has a long pre invasive state, accessibility of screening procedures and effectual management of pre invasive lesions. Earlier diagnosis of CIN is compulsory. Colposcopy can be used as screening tool for detecting pre cancerous lesions.

\section{Conflict of interest: None. Disclaimer: Nil.}

\section{References}

1.World Health Organization. WHO guidance note comprehensive cervical cancer prevention and control: a healthier future for girls and women. Zeneva: WHO; 2013.

2.Jones HW, Rock JA, editors. Te Linde's operative gynecology. Philadelphia: Lippincott Williams \& Wilkins; 2015.

3.Koss LG, Melamed MR, editors. Koss' diagnostic cytology and its histopathologic bases. Philadelphia: Lippincott Williams \& Wilkins; 2006.

4.Dutta DC. Text Book of Obstetrics: Including Perinatology and Contraception. New Delhi: Central book agency; 2004. 
The New Indian Journal of OBGYN. 2019 (January-June); 5(2)

5.Reagan JW, Seidemann IL, Saracusa Y. The cellular morphology of carcinoma in situ and dysplasia or atypical hyperplasia of the uterine cervix. Cancer. 1953 M; 6(2): 22435.

6.Raychaudhuri S, Mandal S. Current status of knowledge, attitude and practice (KAP) and screening for cervical cancer in countries at different levels of development. Asian Pacific Journal of Cancer Prevention. 2012; 13(9): 4221-7.

7.Miller AB, Sankaranarayanan R, Bosch FX, Sepulveda C. Can screening for cervical cancer be improved, especially in developing countries? International journal of cancer. 2003;107(3): 337- 40 .

8.Kushtagi P, Fernandes P. Significance of persistent inflammatory cervical smears in sexually active women of reproductive age. J Obstet Gynaecol India. 2002; 52(1): 124 6.

9.Dhaubhadel P, Vaidya A, Choudhary P. Early detection of precursors of cervical cancer with cervical cytology and visual inspection of cervix with acetic Acid. Journal of the Nepal Medical Association. 2008; 47(170):71-6

10. Shalini R, Amita S, Neera MA. How alarming is postcoital bleeding - a cytologic, colposcopic and histopathologic evaluation. Gynecologic and obstetric investigation. 1998; 45(3): 205-8.
11.Mhaske M, Jawadekar SJ, Saundale SG. Study of association of some risk factors \& cervical dysplasia/cancer among rural women. National Journal of Community Medicine. 2011; 2(2): 209-12.

12.Stern E. Epidemiology of dysplasia. Obstetrical \& gynecological survey. 1969; 24(7): 711-23.

13. Wright Jr TC, Cox JT, Massad LS, Twiggs LB, Wilkinson EJ. 2001 consensus guidelines for the management of women with cervical cytological abnormalities. JAMA. 2002; 287(16): 2120-9.

14.Olaniyan OB. Validity of colposcopy in the diagnosis of early cervical neoplasia: a review. African Journal of Reproductive Health. 2002; 1: 59-69.

\footnotetext{
Nagajan Bhadarka ${ }^{1}$, Nimish Pandya ${ }^{2}$, Satyam Joshi ${ }^{3}$

${ }^{1}$ Associate Professor, Department of Obstetrics \& Gynaecology; ${ }^{2}$ Professor \& HOD, Department of Obstetrics \& Gynaecology, Gujarat Adani Institute of Medical Science, Bhuj, Kutch, Gujarat, India; ${ }^{3}$ BDS, Pacific Dental College and Hospital, Udaipur, Rajasthan
} 